\section{Aleitamento materno na primeira hora de vida em um Hospital Amigo da Criança: prevalência, fatores associados e razões para sua não ocorrência}

\section{Maternal breastfeeding in the first hour of life at a Child-Friendly Hospital: prevalence, associated factors and reasons for its non- occurrence}

Mércia Natália Macêdo Belo 1

Pedro Tadeu Álvares Costa Caminha de Azevedo2 Marcela Patricia Macêdo Belo 3

Vilneide Maria Santos Braga Diégues Serva 4 Malaquias Batista Filho 5

José Natal Figueiroa 6

Maria de Fátima Costa Caminha 7

1 Departamento de Enfermagem. Universidade Federal de Pernambuco. Recife, PE, Brasil.

2 Graduação em Medicina. Faculdade Pernambucana de Saúde. Recife, PE, Brasil.

3 Departamento de Saúde da Criança e do Adolescente. Universidade Federal de Pernambuco. Recife, PE, Brasil.

4-7 Instituto de Medicina Integral Prof. Fernando Figueira. Rua dos Coelhos, 300. Boa Vista. Recife, PE, Brasil. CEP: 50.070-550.

E-mail: fatimacaminha@imip.org.br

\section{Resumo}

Objetivos: identificar a prevalência do aleita-

Objectives: to identify the prevalence of maternal breastfeeding in the first hour of life, associated factors and reasons for its non-occurrence at a childfriendly hospital.

Methods: a cross-sectional study was carried out with 562 mothers and newborns. The data were obtained between October and November 2011 using interview forms and medical records. The Poisson model was adjusted for analysis of the prevalence of maternal breastfeeding in the first hour of life according to exposure variables.

Results: the prevalence of maternal breastfeeding in the first hour of life was $31 \%$. Only normal birth remained in the final model, with a prevalence rate $27 \%$ higher than caesarian birth $(p=0.020)$. The reasons why 388 children were not breastfed in the first hour of life were classified as: problems with the health of the child $(328,84.5 \%)$, problems with the health of the mother $(241,62.1 \%)$ and delayed result of anti-HIV test (199, 51.2\%); in the case of eleven newborns (2.8\%) no reason was found.

Conclusions: the results reported are still well below the World Health Organization (WHO) recommendations and this can largely be attributed to the specific conditions of a tertiary level hospital, which predominantly caters for medium- and high-risk obstetric patients.

Key words Breast feeding, Maternal and child health, Postpartum period mento materno na primeira hora de vida, os fatores associados e as razões para sua não ocorrência em um Hospital Amigo da Criança.

Métodos: estudo transversal com 562 mães $e$ recém-nascidos. Os dados foram obtidos entre outubro a novembro de 2011 mediante formulários de entrevista e consulta a prontuários. Modelo de Poisson foi ajustado para analisar a prevalência do aleitamento materno na primeira hora de vida em função das variáveis de exposição.

Resultados: a prevalência do aleitamento materno na primeira hora de vida foi de 31\%. Apenas o parto normal permaneceu no modelo final, apresentando razão de prevalência de $27 \%$ a mais em relação ao parto cesáreo $(p=0,020)$. As razões para que 388 crianças não tenham sido amamentadas na primeira hora de vida foram classificadas em: problemas de saúde da criança (328, 84,5\%), da mãe (241, 62,1\%) e atraso no resultado do teste rápido anti-HIV (199, 51,2\%), 11 (2,8\%) não apresentaram nenhuma justificativa.

Conclusões: os resultados relatados ainda estão bem abaixo das recomendações da Organização Mundial da Saúde (OMS), o que em grande parte pode ser atribuido às condições próprias de um hospital de nível terciário, cuja demanda é constituida predominantemente por casos de pacientes de médio e elevado risco obstétrico.

Palavras-chave Aleitamento materno, Saúde materno-infantil, Período pós-parto 


\section{Introdução}

Após o ciclo de difusão desordenada do desmame precoce em escala mundial, como prática globalizada decorrente da economia de mercado, 1 a "redescoberta" da amamentação como uma alternativa de importância prioritária para a promoção de saúde das crianças passou a figurar como uma estratégia universal de redução da morbimortalidade na infância. ${ }^{2-4}$ É oportuno refletir que os efeitos protetores da amamentação se estendem à mãe e se ampliam para todo o ciclo vital, incluindo a redução significativa do risco de doenças crônicas não transmissíveis e das suas complicações. ${ }^{5,6}$

Possivelmente, uma das abordagens mais inovadoras dos estudos sobre o aleitamento materno foi a descoberta que a amamentação a partir do primeiro dia de vida pode evitar $16 \%$ das mortes neonatais. Essa taxa pode se elevar para $22 \%$ se a amamentação for antecipada para a primeira hora após o parto, ${ }^{7}$ representando um incremento considerável na diminuição dos riscos de morte numa etapa crucial para a sobrevivência e desenvolvimento da criança. ${ }^{1}$ Assim, um estudo multicêntrico envolvendo 67 países evidenciou que os recémnascidos de países situados no tercil mais elevado de aleitamento materno tiveram uma taxa de mortalidade neonatal $24 \%$ menor mesmo após ajuste para fatores de confundimento da correlação ecológica. ${ }^{8}$

Estas evidências justificam a recomendação de se antecipar ao máximo possível o início da amamentação, como uma forma de reduzir o absurdo dife-rencial que contrasta, por exemplo, a mortalidade neonatal de $1 \%$ no Japão, enquanto que na Somália atinge $50 \%$. Entre esses extremos se posicionam os 52 países avaliados que compõem a comunidade de nações independentes, com a particularidade de que os que fazem parte do bloco dos países menos desenvolvidos se aproximam mais do "padrão Somália" que do japonês ou islandês. 9

No que concerne à prática da amamentação em crianças brasileiras, dados da II Pesquisa de Prevalência de Aleitamento Materno nas Capitais e Distrito Federal revelam que $67,7 \%$ das crianças foram amamentadas na primeira hora de vida. 10 Estudos isolados apontam índices menores como o de Silveira et al. ${ }^{11}$ ao relatarem que $35,5 \%$ das mães residentes em Pelotas, RS, amamentaram na primeira hora pós-parto, enquanto numa avaliação realizada em maternidades do Rio de Janeiro observou-se que apenas $16 \%$ das parturientes o fizeram. 12

$\mathrm{O}$ aleitamento materno na primeira hora de vida é considerado um indicador de excelência da amamentação. Neste sentido, a Organização Mundial da Saúde (OMS) classifica os percentuais de adesão ao aleitamento na primeira hora para mães e recémnascidos sadios entre 0 e $29 \%$ como "muito ruim", 30 a $49 \%$ "ruim", 50 a $89 \%$ "bom" e de 90 a $100 \%$ "muito bom". 13

A OMS e o Fundo das Nações Unidas para a Infância (UNICEF) recomendam colocar os recémnascidos em contato com suas mães imediatamente após o parto, durante pelo menos uma hora e encorajá-las a reconhecerem quando estejam prontos para mamar, oferecendo ajuda, se necessária. ${ }^{14} \mathrm{O}$ estímulo à amamentação por meio do contato mãe-filho (também chamado pele a pele) na sala de parto corresponde ao Passo 4 da Iniciativa Hospital Amigo da Criança (IHAC). 15,16

Tendo em vista a importância desta estratégia de baixo custo para prevenção de mortes neonatais, este estudo teve como objetivo identificar a prevalência do aleitamento na primeira hora de vida, os fatores associados a sua prática e as razões para sua não ocorrência em um hospital Amigo da Criança no nordeste brasileiro, sendo fundamental para a adoção de medidas voltadas para a melhoria dessa prática, principalmente nas instituições de referência.

\section{Métodos}

Estudo transversal, realizado no alojamento conjunto do Instituto de Medicina Integral Prof. Fernando Figueira (IMIP), em Recife, Pernambuco, um hospital regional de referência do Ministério da Saúde (MS) para a assistência materno-infantil que atende, prioritariamente, mulheres com gestações de médio e alto risco. A unidade hospitalar foi a primeira a receber a distinção de "Amigo da Criança" no Brasil, por seu desempenho na promoção do aleitamento materno.

A coleta de dados realizou-se entre outubro e novembro de 2011. No alojamento conjunto do IMIP são admitidas mensalmente cerca de 320 mães e recém-nascidos procedentes da maternidade do próprio hospital. $\mathrm{O}$ alojamento conjunto funciona como espaço facilitador dos cuidados maternos no pós-parto, permitindo que o recém-nascido permaneça ao lado da mãe continuamente. Esse ambiente favorece a promoção da amamentação, bem como o fortalecimento do elo mãe-filho.

O tamanho da amostra foi calculado no módulo STATCALC do EPI-Info 6.04, adotando-se um nível de confiança de $95 \%$ e um erro de estimação de 3\%, admitindo-se prevalência de $16 \%$ para o aleitamento materno na primeira hora após o nascimento, 12 resultando em uma estimativa amostral de 573 mães e crianças.

O processo de amostragem foi consecutivo, sendo arrolada para o estudo toda a casuística 
acessível no período de tempo estabelecido para que se chegasse ao número calculado como estimativa amostral. Os critérios de exclusão foram a contraindicação para o aleitamento materno, ocorrência do parto fora do hospital, gemelaridade, menores de idade desacompanhadas de responsável legal, alta hospitalar fora do horário regular do serviço e a negativa a participar do estudo.

Considerou-se como desfecho a oferta do seio materno para amamentação do neonato pela puérpera até 60 minutos após o nascimento, sendo obtido pelos próprios pesquisadores, através de entrevista com a mãe da criança, nas primeiras 24 horas após a admissão no alojamento conjunto. As variáveis explanatórias (características amostrais) foram biológicas (idade materna), sociais (raça/cor, estado civil, anos de estudo, renda per capita) demográficos e obstétricos (número de consultas no pré-natal, orientações no pré-natal sobre aleitamento materno na primeira hora, exame clínico da mama no prénatal e paridade). Dados complementares, relativos peso ao nascer, sexo, raça/cor, tipo de parto, idade gestacional, Apgar no $1^{\circ}$ e no $5^{\circ}$ minutos, foram extraídos dos prontuários das mães e crianças.

As características amostrais foram obtidas através de frequências absolutas e relativas compondo 14 variáveis de informações demográficas, socioeconômicas, clínicas e de acesso à assistência por parte das mães e dos recém-nascidos. A análise dos fatores associados ao aleitamento materno na primeira hora de vida foi realizada através da regressão multivariada de Poisson, estimando-se as razões de prevalência (RP) brutas, ajustadas e os respectivos intervalos de confiança de 95\%. Participaram da análise multivariada as variáveis que na análise univariada apresentaram valor $p<20 \%$. Para definir o modelo final foi considerando o nível de significância $<5 \%$. As análises foram realizadas no software Stata 12.1 SE.

O projeto de estudo foi aprovado pelo Comitê de Ética em Pesquisa em Seres Humanos do Instituto de Medicina Integral Prof. Fernando Figueira sob o protocolo $\mathrm{n}^{\mathrm{o}} 2568-11$.

\section{Resultados}

Durante os dois meses da coleta de dados foram internadas no alojamento conjunto do IMIP 686 puérperas que de acordo com os critérios de elegibilidade. Destas foram excluídas 124, das quais 27 por contraindicação para o aleitamento materno (26 HIV positivo e uma por infecção pelo Vírus Linfotrófico Humano de Células T - HTLV), quatro pelo parto não ter ocorrido na Instituição, 12 por gemelaridade, 47 menores de idade desacompanhadas de respon- sável legal, 26 por terem recebido alta hospitalar fora do horário regular do serviço e oito por se negarem a participar do estudo. Desta forma, a amostra final foi constituída de 562 mães e 562 recém-nascidos.

Dos 562 recém-nascidos ( $\mathrm{RN})$ estudados, apenas 174 (31\%) mamaram na primeira hora de vida. A Tabela 1 mostra que cerca de $70 \%$ das mães tinham idade entre 20 e 35 anos e $73 \%$ haviam concluído nove anos ou mais de estudos formais. Sobre os dados obstétricos, observou-se que $98 \%$ das mães realizaram consultas pré-natais na rede pública de saúde, pouco mais da metade receberam informações durante o pré-natal, sobre a importância do aleitamento na primeira hora de vida da criança. O parto vaginal foi predominante $(60,0 \%)$. Ademais, $65,8 \%$ pertenciam a famílias com renda per capita familiar abaixo de 0,5 salário mínimo e $72,1 \%$ receberam seis ou mais consultas pré-natais. Outras informações que caracterizam a amostra encontram-se detalhadas na Tabela 1.

A Tabela 2 apresenta os resultados das análises univariadas e multivariadas dos fatores associados ao aleitamento materno na primeira hora de vida. $\mathrm{Na}$ análise univariada, as variáveis Apgar no primeiro minuto, paridade, tipo de parto, peso ao nascer, anos de estudos, orientações no pré-natal sobre aleitamento na primeira hora e prematuridade apresentaram valores de $p<0,20$, credenciando-se assim para a etapa da análise multivariada. No entanto, após os ajustes, apenas a variável parto vaginal apresentou associação significante com a amamentação na primeira hora de vida $(p=0,020)$.

Das 388 mães que não amamentaram na primeira hora de vida, cinco $(1,3 \%)$ não souberam informar quando a amamentação foi iniciada. Das 383 que informaram esse dado, 97 (25,3\%) iniciaram a amamentação até duas horas após o parto, 41 $(10,7 \%)$ até três horas e $245(64,0 \%)$ após três horas de nascimento.

Dentre as 388 crianças não amamentadas na primeira hora de vida, foram destacadas três principais razões para a não ocorrência da amamentação: a) Problemas de saúde $(328 / 388,84,5 \%)$, desses 328 recém-nascidos, $222(67,7 \%)$ tratavam-se de algum grau de desconforto respiratório ao nascimento; b) Problemas de saúde materna $(241 / 388,62,1 \%)$, dessas 241 mães, 94 (39,0\%) tiveram pré-eclâmpsia grave durante o período gravídico-puerperal; c) Atraso no resultado do teste rápido anti-HIV (199/388, 51,2\%). Ressalta-se que em cada caso pode ter ocorrido mais de uma razão. Onze crianças não apresentaram nenhuma justificativa para não serem amamentadas e doze tiveram como razão apenas o atraso no resultado do teste rápido antiHIV. 
Tabela 1

Características amostrais das mães e recém-nascidos internados em alojamento conjunto de um hospital regional de referência para a atenção materno-infantil, Recife, Pernambuco, 2011.

\begin{tabular}{|c|c|c|}
\hline Variáveis/Categorias & N (562) & $\%$ \\
\hline \multicolumn{3}{|c|}{ Características socioeconômicas e demográficas } \\
\hline \multicolumn{3}{|l|}{ Idade materna (anos) } \\
\hline$<20$ & 116 & 20,6 \\
\hline 20 a 35 & 385 & 68,5 \\
\hline$\geq 36$ & 61 & 10,9 \\
\hline \multicolumn{3}{|l|}{ Raça/Cor } \\
\hline Branca & 102 & 18,1 \\
\hline Negra & 59 & 10,5 \\
\hline Parda/amarela & 401 & 71,4 \\
\hline \multicolumn{3}{|l|}{ Estado civil } \\
\hline Solteira & 137 & 24,4 \\
\hline Casada/União consensual & 425 & 75,6 \\
\hline \multicolumn{3}{|l|}{ Anos de estudo } \\
\hline$\leq 4$ & 17 & 3,0 \\
\hline 5 a 8 & 135 & 24,0 \\
\hline$\geq 9$ & 410 & 73,0 \\
\hline \multicolumn{3}{|l|}{ Renda per capita* $(\mathrm{SM}) * *$} \\
\hline$<0,5$ & 375 & 65,8 \\
\hline$\geq 0,5$ & 157 & 28,0 \\
\hline \multicolumn{3}{|c|}{ Precedentes obstétricos e relativos a serviços de saúde } \\
\hline \multicolumn{3}{|c|}{ Número de consultas no pré-natal } \\
\hline$<6$ & 157 & 27,9 \\
\hline$\geq 6$ & 405 & 72,1 \\
\hline \multicolumn{3}{|c|}{ Orientações no pré-natal sobre aleitamento materno na primeira hora } \\
\hline Sim & 310 & 55,2 \\
\hline Não & 252 & 44,8 \\
\hline \multicolumn{3}{|l|}{ Exame da mama no pré-natal } \\
\hline Sim & 373 & 66,4 \\
\hline Não & 189 & 33,6 \\
\hline \multicolumn{3}{|l|}{ Tipo de parto } \\
\hline Vaginal & 347 & 60,0 \\
\hline Cesáreo & 215 & 40,0 \\
\hline \multicolumn{3}{|l|}{ Paridade } \\
\hline Primípara & 278 & 49,5 \\
\hline Multípara & 284 & 50,5 \\
\hline \multicolumn{3}{|c|}{ Características biológicas referentes às crianças } \\
\hline \multicolumn{3}{|l|}{ Prematuridade } \\
\hline Sim & 93 & 16,5 \\
\hline Não & 469 & 83,5 \\
\hline \multicolumn{3}{|l|}{ Sexo } \\
\hline Masculino & 276 & 49,1 \\
\hline Feminino & 286 & 50,9 \\
\hline \multicolumn{3}{|l|}{ Apgar no $1^{\circ}$ minuto } \\
\hline 0 a 6 & 46 & 8,2 \\
\hline 7 a 10 & 516 & 91,8 \\
\hline \multicolumn{3}{|l|}{ Peso ao nascer $(\mathrm{g})$} \\
\hline$<2500$ & 71 & 12,6 \\
\hline$\geq 2500$ & 491 & 87,4 \\
\hline
\end{tabular}

*Trinta mães se recusaram a informar o valor da renda familiar; * $\mathrm{SM}=$ salário mínimo $(\mathrm{R} \$ 545,00)$. 
Regressão de Poisson para a relação entre amamentação na primeira hora de vida e variáveis clínicas e sociodemográficas, em um hospital regional de referência para a atenção materno-infantil, Recife, Pernambuco, 2011.

\begin{tabular}{|c|c|c|c|c|c|c|c|}
\hline \multirow[t]{2}{*}{ Variáveis/Categorias } & \multirow[t]{2}{*}{ Total } & \multicolumn{6}{|c|}{ Amamentação na primeira hora de vida } \\
\hline & & $\mathrm{n}$ & $\%$ & $\mathrm{RP}_{\text {bruta }}(\mathrm{IC} 95 \%)$ & $p$ & $\mathrm{RP}_{\text {ajustada }}(\mathrm{IC95 \% )}$ & $p$ \\
\hline Idade materna (anos) & & & & & 0,484 & - & - \\
\hline$<20$ & 116 & 39 & 33,6 & 1,0 & & & \\
\hline 20 a 35 & 385 & 120 & 31,2 & $0,93(0,69-1,25)$ & & & \\
\hline$\geq 36$ & 61 & 15 & 24,6 & $0,73(0,44-1,22)$ & & & \\
\hline Raça/Cor materna & & & & & 0,760 & - & - \\
\hline Branca & 102 & 29 & 28,4 & $0,84(0,52-1,34)$ & & & \\
\hline Negra & 59 & 20 & 33,9 & 1,0 & & & \\
\hline Parda/Amarela & 401 & 125 & 31,2 & $0,92(0,62-1,35)$ & & & \\
\hline Estado civil & & & & & 0,441 & - & - \\
\hline Solteira & 137 & 46 & 33,6 & 1,0 & & & \\
\hline Casada/ União consensual & 425 & 128 & 30,1 & $0,90(0,68-1,18)$ & & & \\
\hline Anos de estudo & & & & & 0,199 & & 0,094 \\
\hline$\leq 4$ & 17 & 5 & 29,4 & $0,79(0,37-1,71)$ & & $0,86(0,40-1,86)$ & \\
\hline 5 a 8 & 135 & 50 & 37,0 & 1,0 & & 1,0 & \\
\hline$\geq 9$ & 410 & 119 & 29,0 & $0,78(0,60-1,02)$ & & $0,75(0,57-0,97)$ & \\
\hline Renda per capita familiar (SM)** & & & & & 0,287 & - & - \\
\hline$<0,5$ & 375 & 123 & 32,8 & 1,0 & & & \\
\hline$\geq 0,5$ & 181 & 44 & 28,0 & $0,85(0,64-1,14)$ & & & \\
\hline Número de consultas no pré- & & & & & 0,132 & & 0,122 \\
\hline natal & & & & & & $0,79(0,59-1,06)$ & \\
\hline$<6$ & 157 & 41 & 26,1 & $0,79(0,59-1,07)$ & & 1,0 & \\
\hline$\geq 6$ & 405 & 133 & 32,8 & 1,0 & & & \\
\hline Orientação sobre amamentação & & & & & 0,069 & & 0,178 \\
\hline na $1^{\text {a }}$ hora no pré-natal & 310 & 106 & 34,2 & 1,0 & & & \\
\hline $\operatorname{Sim}$ & 252 & 68 & 27,0 & $0,79(0,61-1,02)$ & & 1,0 & \\
\hline Não & & & & & & $0,83(0,64-1,1)$ & - \\
\hline Exame da mama no pré-natal & & & & & 0,388 & - & \\
\hline $\operatorname{Sim}$ & 373 & 120 & 32,2 & 1,0 & & & \\
\hline Não & 189 & 54 & 28,6 & $0,89(0,68-1,16)$ & & & \\
\hline Tipo de parto & & & & & 0,021 & & 0,020 \\
\hline Vaginal & 347 & 120 & 34,6 & 1,0 & & 1,0 & \\
\hline Cesáreo & 215 & 54 & 25,1 & $0,73(0,55-0,95)$ & & $0,79(0,55-0,95)$ & \\
\hline Paridade & & & & & 0,199 & & 0,332 \\
\hline Primípara & 278 & 79 & 28,4 & $0,85(0,66-1,09)$ & & $0,88(0,68-1,13)$ & \\
\hline Multípara & 284 & 95 & 33,5 & 1,0 & & 1,0 & \\
\hline Idade gestacional (semanas) & & & & & 0,070 & & 0,476 \\
\hline$<37$ & 93 & 21 & 22,6 & $0,69(0,46-1,03)$ & & $0,84(0,53-1,35)$ & \\
\hline$\geq 37$ & 469 & 153 & 32,6 & 1,0 & & 1,0 & \\
\hline Sexo & & & & & 0,233 & - & - \\
\hline Masculino & 276 & 92 & 33,3 & 1,0 & & & \\
\hline Feminino & 286 & 82 & 28,7 & $0,86(0,67-1,10)$ & & & \\
\hline Apgar no $1^{\circ}$ minuto & & & & & 0,061 & & 0,127 \\
\hline 0 a 6 & 46 & 8 & 17,4 & $0,54(0,28-1,03)$ & & $0,60(0,32-1,15)$ & \\
\hline 7 a 10 & 516 & 166 & 32,2 & 1,0 & & 1,0 & \\
\hline Peso de nascimento $(\mathrm{g})$ & & & & & 0,073 & & 0,108 \\
\hline$<2500$ & 71 & 15 & 21,1 & $0,65(0,41-1,04)$ & & $0,68(0,43-1,09)$ & \\
\hline$\geq 2500$ & 491 & 159 & 32,4 & 1,0 & & 1,0 & \\
\hline
\end{tabular}

SM = salário mínimo na época da pesquisa $(R \$ 545,00)$. 


\section{Discussão}

Tratando-se de uma meta de saúde de recomendação ainda recente, é compreensível que a adoção do aleitamento materno na primeira hora após o nascimento não tenha alcançado os resultados esperados. Como se reconhece através da história e da antropologia da alimentação, as condutas relacionadas com o aleitamento materno variaram consideravelmente no transcurso dos séculos, na medida em que representam comportamentos condicionados por razões culturais, políticas, econômicas e crenças religiosas, determinando valores e condutas coletivas. $1,4,9$

A proposta de se iniciar o aleitamento materno logo após o nascimento, segundo um informe recente da UNICEF, ${ }^{9}$ relatado de um conjunto de 108 países avaliados, indica que $13,9 \%$ se enquadram na classificação "muito ruim", $35,2 \%$ se agrupam na categoria "ruim", seguindo-se $50,9 \%$ dos países que ocupam uma classificação considerada boa. Finalmente, nenhum dos países avaliados atingiu a categoria "muito boa", ou seja, um patamar igual ou acima de $90 \%$.

$\mathrm{Na}$ realidade, sendo um indicador de excelência das práticas de aleitamento materno como um dos condicionantes preditivos da sobrevivência e desenvolvimento das crianças nos primeiros meses de vida, a amamentação na primeira hora após o nascimento ainda constitui uma meta longe de ser alcançada. É, portanto, dentro desta lógica preliminar que os dados analisados em nosso estudo devem ser contextualizados.

De modo geral, os valores de prevalência nacionais, incluindo capitais dos Estados de todas as regiões do Brasil, além do Distrito Federal, são bem mais homogêneos que os dados apresentados no cenário internacional. ${ }^{10}$ Porém nas sete capitais da Região Norte as prevalências variaram entre $64,3 \%$ e 79,6\%, enquanto nas nove capitais do Nordeste se situaram entre $58,5 \%$ e $83,5 \%$. Variações da mesma ordem foram encontradas no Centro-Oeste, Sudeste e Sul do Brasil. Para o conjunto do País, a prevalência encontrada foi de $67,7 \% .10$ Portanto, como se pode verificar, os cenários de prevalência do aleitamento materno na primeira hora de vida no território nacional são bem melhores que os retratados para a maternidade aqui avaliada.

Partindo destas duas perspectivas, evidencia-se que a situação do caso do hospital de referência aqui avaliado se enquadraria mais no contexto externo que no cenário das capitais brasileiras. Cabe, de antemão, uma reflexão preliminar: a diversidade de procedimentos metodológicos que dificultam a comparabilidade dos resultados e as inferências que poderiam derivar de suas comparações. Contudo, como a instituição estudada é referência regional do MS para a assistência materno-infantil, se destacando por seus esforços para a promoção do aleitamento em nível de ações básicas de saúde, de ambulatórios especializados em pediatria e obstetrícia e, principalmente, na assistência ao parto e aos recémnatos, confere uma situação instigante para a análise dos resultados aqui discutidos.

Deve-se reconhecer que, tratando-se de um serviço de nível terciário, com elevada demanda de casos obstétricos complicados (médio e elevado risco), já se estabelece um contexto de dificuldades a serem enfrentadas para a prática da amamentação na primeira hora de vida. Assim, fica evidenciado que o parto cesáreo representado por $40 \%$ das mulheres deste estudo, provavelmente foi um dos fatores adversos para a prevalência encontrada, podendo, ainda, explicar e até justificar a permanência do tipo de parto no modelo final. Isto concorda com a observação de Boccolini et al. 17 no Rio de Janeiro, verificando que, nos casos de parto vaginal, $22,4 \%$ dos recém-nascidos iniciaram a amamentação na primeira hora de vida, enquanto nos casos cirúrgicos esta proporção caiu para apenas $5,8 \%(p<0,001)$.

Dentre os fatores maternos, a pré-eclâmpsia grave foi a principal limitação para a não iniciação da amamentação na primeira hora após o parto. Trata-se, por sinal, de uma das causas mais importantes de internação em unidades de terapia obstétrica intensiva.18 Além disso, distúrbios hipertensivos na gestação elevam substancialmente a morbidade dos recém-nascidos, com a necessidade de cuidados especiais. ${ }^{19}$ Ressalta-se que a amamentação na primeira hora de vida abrange somente mães e recém-nascidos saudáveis. ${ }^{14}$

Em relação aos problemas de saúde da criança, o desconforto respiratório foi a principal razão para a não ocorrência da amamentação na primeira hora de vida. Em São Paulo, Pillegi et al. ${ }^{20}$ classificaram o desconforto respiratório como uma contraindicação para a amamentação. Entretanto, a dificuldade respiratória após o nascimento pode representar tanto uma condição benigna, como retardo na adaptação cardiorrespiratória e pode, ainda, indicar quadros infecciosos. Assim, alcançar o diagnóstico correto torna-se um desafio. 21

O MS contraindica a amamentação apenas em situações muito específicas, sendo a infecção pelo vírus da imunodeficiência humana (HIV) uma delas. ${ }^{22} \mathrm{Na}$ perspectiva de prevenção da transmissão vertical o teste rápido anti-HIV constitui um exame simples e eficiente que apresenta resultado em 
menos de trinta minutos, o que possibilita intervenção profilática de emergência nos casos reagentes. ${ }^{23,24}$ Contudo, neste estudo verificou-se que cerca da metade das mães que não amamentaram seus filhos logo após o nascimento tiveram seus resultados atrasados em mais de uma hora pós-parto. Problemas com a obtenção do resultado do teste rápido anti-HIV também foram encontrados no estudo de Oliveira et al.,25 no qual das 944 mães submetidas ao teste, apenas $15,6 \%$ souberam do resultado antes do parto.

Das crianças estudadas, onze não apresentaram nenhuma razão para a não ocorrência da amamentação na primeira hora de vida, representando, dessa forma, casos de oportunidades perdidas para essa prática. Ademais, doze crianças e suas respectivas mães, dentre aquelas com atraso no resultado do teste rápido anti-HIV, não tinham razões clínicas aceitáveis para não serem amamentadas, o que caracteriza situações que poderiam ter sido prevenidas.

Conclui-se que a amamentação na primeira hora de vida representou uma frequência aquém do

\section{Referências}

1. Caminha MFC, Serva VB, Arruda IKG, Batista Filho M. Aspectos históricos, científicos, socioeconômicos e institucionais do aleitamento materno. Rev Bras Saúde Matern Infant. 2010; 10: 25-37.

2. Victora CG, Huttly SR, Fuchs SC, Nobre LC, Barros FC. Deaths due to dysentery, acute and persistent diarrhoea among Brazilian infants. Acta Paediatr. 1992; 381: 7-11.

3. Boccolini CS, Carvalho ML, Oliveira MIC, Boccolini PMM. O papel do aleitamento materno na redução das hospitalizações por pneumonia em crianças brasileiras menores de 1 ano. J Pediatr. 2011; 87: 399-404.

4. World Health Organization (WHO), Regional Office for the Americas. Indicators for Assessing Infant and Young Child Feeding Practices, Washington; 2008. [acesso em 10 mar 2012]. Disponível em: http://whqlibdoc.who.int/publications/2008/9789241596664_eng.pdf.)

5. Alves JGB, Figueira F. Doenças do adulto com raízes na infância. 2 ed. Recife: Medbook; 2010.

6. Fewtrell MS, Morgan JB, Duggan C, Gunnlaugsson G, Hibberd PL, Lucas A, Kleinman RE. Optimal duration of exclusive breastfeeding: what is the evidence to support current recommendations? Am J Clin Nutr. 2007; 85: 635S$8 \mathrm{~S}$.

7. Edmond KM, Zandoh C, Quigley MA, Amenga-Etego S, Owusu-Agyei S, Kirkwood BR. Delayed Breastfeeding Initiation Increases Risk of Neonatal Mortality. Pediatrics. 2006; 117: 380-6.

8. Boccolini CS, Carvalho ML, Oliveira MI, Pérez-Escamilla R. Breastfeeding during the first hour of life and neonatal mortality. J Pediatr. 2013; 89: 131-6. recomendado pela OMS. Na realidade, o que se deve postular é a maximização da prática nos casos possíveis, o que significaria a admissão de uma faixa de tolerância para as exceções tecnicamente justificadas. Isto inclui a reconsideração de resgatar a prática do aleitamento materno na primeira hora mesmo em muitos casos de intercorrências de saúde da mãe e do concepto. Por outro lado, devem-se controlar fatores preveníveis, como o atraso na liberação do resultado do teste rápido anti-HIV, bem como estimular o parto vaginal, nos casos em que as condições de saúde da mãe e da criança permitirem, como fatores que podem favorecer o início do aleitamento materno na primeira hora após o parto.

Sugere-se que os resultados e suas análises possam representar o pretexto para uma discussão interna com os "staffs" técnicos e administrativos dos hospitais/maternidades de nível terciário que, desafiados pelo atendimento de casos de médio e elevado risco obstétrico, possam buscar estratégias alternativas ou complementares para melhorar seu desempenho, no que concerne a difusão da prática da amamentação na primeira hora de vida.

9. UNICEF (Fundo das Nações Unidas para a Infância). Situação mundial da infância: 2013. Crianças com deficiência. Brasília (DF): UNICEF; 2013. [acesso em 11 nov 2013]. Disponível em: http://www.unicef.org/brazil/pt/ PT_SOWC2013.pdf

10. Venancio SI, Escuder MML, Saldiva SRDM, Giuliani ERJ. A prática do aleitamento materno nas capitais brasileiras e Distrito Federal: situação atual e avanços. J Pediatr. 2010; 86: $317-24$

11. Silveira RB, Albernaz E, Zuccheto LM. Fatores associados ao início da amamentação em uma cidade do sul do Brasil. Rev Bras Saúde Matern Infant. 2008; 8: 35-43.

12. Boccolini CS, Carvalho ML, Oliveira MIC, Vasconcellos AGG. Fatores associados à amamentação na primeira hora de vida. Rev Saúde Pública. 2011; 45: 69-76.

13. WHO (World Health Organization). Infant and young child feeding: a tool for assessing national practices, policies and programmes. Geneva; 2003. [acesso em 15 jul 2012]. Disponível em: http:/www.who.int/nutrition/publications/ infantfeeding/inf_assess_nnpp_eng.pdf.

14. OMS/UNICEF (Organização Mundial da Saúde/ Fundo das Nações Unidas para a Infância). Iniciativa Hospital Amigo da Criança: revista, atualizada e ampliada para o cuidado integrado. Módulo 3 - Promovendo e Incentivando a Amamentação em um Hospital Amigo da Criança: Curso de 20 horas para Equipes de Maternidade. Série A. Normas e Manuais Técnicos. Brasília; 2009.

15. Araújo MFM, Otto AFN, Schmitz BAS. Primeira avaliação do cumprimento dos "Dez passos para o sucesso do aleitamento materno" nos hospitais amigos da criança do Brasil. Rev Bras Saúde Matern Infant. 2003; 3: 411-9. 
16. WHO/UNICEF (World Health Organization/United Nations Children's Fund). Baby-friendly hospital initiative: revised, updated and expanded for integrated care. Section 1, Background and implementation. New York; 2009. [acesso em 28 mai 2011]. Disponível em: http://www.unicef.gr/pdfs/BFHI_2009update.pdf

17. Boccolini CS, Carvalho ML, Oliveira MIC, Leal MC, Carvalho MS. Fatores que interferem no tempo entre o nascimento e a primeira mamada. Cad Saúde Pública. 2008; 24: 2681-94.

18. Keizer JL, Zwart JJ, Meerman RH, Harinck BI, Feuth HDM, Roosmalen JV. Obstetric intensive care admissions a 12-year review in a tertiary care centre. Eur J Obstet Gynecol Reprod Biol. 2006; 128: 152-6.

19. Gruslin A, Lemyre B. Pre-eclampsia: fetal assessment and neonatal outcomes. Best Pract Res Clin Obst Gynaecol. 2011; 25: 491-507.

20. Pillegi MC, Policastro A, Abramovici S, Cordioli E, Deutsch AD'A. A amamentação na primeira hora de vida e a tecnologia moderna: prevalência e fatores limitantes. Einstein. 2008; 6: 467-72.

21. Brasil. Ministério da Saúde. Secretaria de Atenção à Saúde, Departamento de Ações Programáticas e Estratégicas. Atenção à saúde do recém-nascido. Brasília, DF; 2011. [acesso em 10 jun 2012]. Disponível em: http://www.fiocruz.br/redeblh/media/arn_v3.pdf.
22. Brasil. Ministério da Saúde. Secretaria de Vigilância em Saúde. Secretaria de Atenção à Saúde. Manual normativo para profissionais de saúde de maternidades - referência para mulheres que não podem amamentar. Brasília, DF; 2005. [acesso em 3 jun 2012]. Disponível em: http://www.ibfan.org.br/documentos/outras/MSmanualHIV eAM2005.pdf.

23. Brasil. Ministério da Saúde. Secretaria de Vigilância em Saúde. Programa Nacional de DST e Aids. Protocolo para a prevenção de transmissão vertical de HIV e sífilis. Brasília, DF; 2007. [acesso em 3 jun 2012]. Disponível em: http://bvsms.saude.gov.br/bvs/publicacoes/protocolo preve ncao_transmissao_verticalhivsifilis_manualbolso.pdf

24. Possolli GT. Fatores associados ao tempo entre o nascimento e o início da amamentação entre mães soronegativas que realizaram teste rápido anti-HIV em Hospitais Amigos da Criança no município do Rio de Janeiro, 2006 [dissertação]. Rio de Janeiro: Escola de Saúde Pública Sérgio Arouca da Fundação Oswaldo Cruz; 2009.

25. Oliveira MIC, Silva KS, Junior SCG, Fonseca VM. Resultado do teste rápido anti-HIV após o parto: uma ameaça à amamentação ao nascimento. Rev Saúde Pública. 2010; 44: 60-9

Recebido em 5 de fevereiro de 2013

Versão final apresentada em 25 de novembro de 2013

Aprovado em 20 de dezembro de 2013 